\title{
Hysteretic magnetoresistance and thermal bistability in a magnetic two-dimensional hole system
}

\author{
Ursula Wurstbauer ${ }^{1,2 \dagger}$, Cezary Śliwa ${ }^{3}$, Dieter Weiss ${ }^{1}$, Tomasz Dietl ${ }^{3,4}$ and Werner Wegscheider ${ }^{1 \star \dagger}$
}

Colossal negative magnetoresistance and the associated field-induced insulator-to-metal transition-the most characteristic features of magnetic semiconductors-are observed in $n$-type rare-earth oxides $^{1}$ and chalcogenides ${ }^{2}$, p-type manganites $^{3}$ and $n$-type ${ }^{4,5}$ and p-type diluted magnetic semiconductors ${ }^{4,6}$, as well as in quantum wells of $n$-type diluted magnetic semiconductors ${ }^{7-9}$. Here, we report on magnetotransport studies of Mn-modulation-doped InAs quantum wells, which reveal an insulator-to-metal transition that is driven by a magnetic field and dependent on bias voltage, with abrupt and hysteretic changes of resistance over several orders of magnitude. These phenomena coexist with the quantized Hall effect in high magnetic fields. We show that the exchange coupling between a hole and the parent $\mathrm{Mn}$ acceptor produces a magnetic anisotropy barrier that shifts the spin relaxation time of the bound hole to a $100 \mathrm{~s}$ range in compressively strained quantum wells. This bistability of the individual Mn acceptors explains the hysteretic behaviour while opening prospects for information storing and processing. At high bias voltage another bistability, caused by the overheating of electrons ${ }^{10}$, gives rise to abrupt resistance jumps.

Molecular beam epitaxy has been used to grow Mn-modulationdoped compressively strained InAs quantum wells (QWs) embedded in InAlAs/InGaAs host material with an In mole fraction of 75\% (for details, see, ref. 11). In this material system Mn substitutes the group III elements (In, $\mathrm{Al}$ or $\mathrm{Ga}$ ), providing a localized spin of $S=5 / 2$ and a hole $e^{6,12,13}$, in contrast to II-VI materials, where $\mathrm{Mn}$ is an isoelectric impurity ${ }^{4,7-9,14}$. Our transport studies have been accomplished on Mn-modulation-doped InAs QW structures depicted schematically in Fig. 1. In the 'normal' sample (Fig. 1b), the Mn doping is done after the InAs/InGaAs layer growth, so that the InAs channel is free of Mn (refs 11,15). In the 'inverted' doped structures (Fig. 1a), the Mn-doped layer is deposited before the growth of the 4-nm-thick InAs channel, which is spaced $7.5 \mathrm{~nm}$ from the Mn-doped layer. This leads to a significant concentration of $\mathrm{Mn}$ in the InAs channel, estimated by secondary ion mass spectroscopy to be $\sim 1 \%$ of the maximum doping concentration ${ }^{11}$.

Figure 1 documents remarkable differences in the transport properties at $1.6 \mathrm{~K}$ of 'normal' and 'inverted' doped samples with comparable two-dimensional hole densities of $p=4.3 \times 10^{10} \mathrm{~cm}^{-2}$ and $p=4.4 \times 10^{10} \mathrm{~cm}^{-2}$, respectively. Both types of sample show temperature- and field-dependent resistances typical for modulation-doped structures, including pronounced

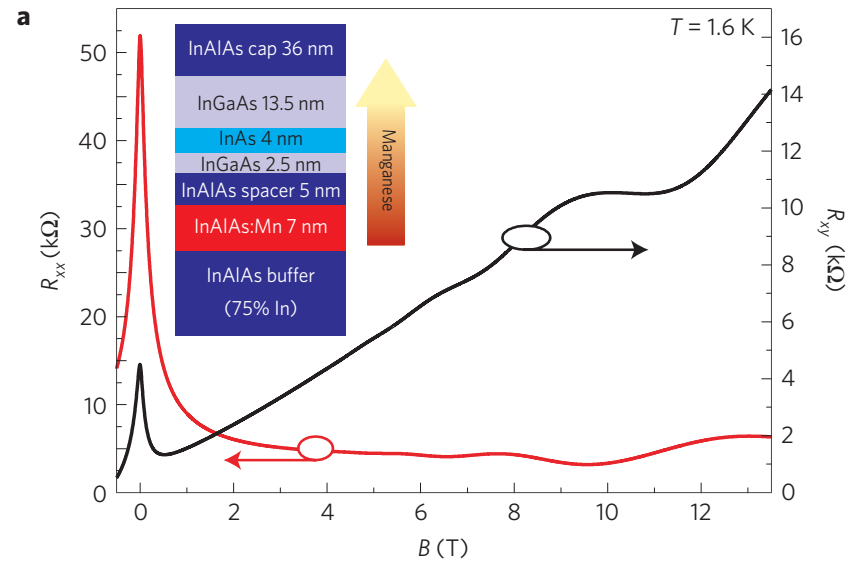

b

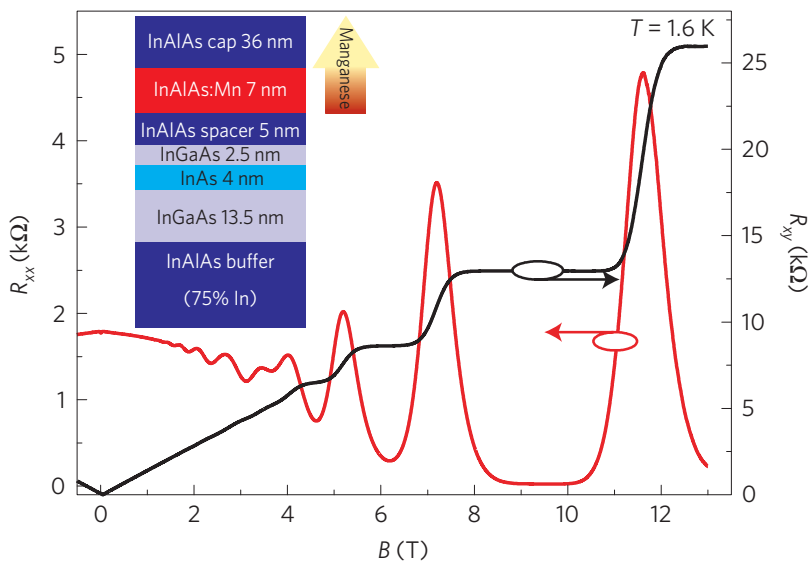

Figure 1 | Results of four-terminal magnetotransport measurements and sample architectures. a,b Longitudinal resistance (red) and Hall resistance (black) at $1.6 \mathrm{~K}$ for inverted (a) and normal (b) modulation-doped QW structures showing well-defined Shubnikov-de Haas oscillations and Hall plateaus. In contrast to the normal-doped QW structure (b), the low-field resistivity increases dramatically for the structure with an inverted doping layer (a), indicating strong hole localization. Insets, Schematic layer sequences of the inverted and normal Mn modulation-doped QW structures. The QW consists of a strain-relaxed InGaAs layer with an asymmetrically embedded strained InAs channel.

\footnotetext{
${ }^{1}$ Institute of Experimental and Applied Physics, University of Regensburg, D-93040 Regensburg, Germany, ${ }^{2}$ Institute of Applied Physics, University of Hamburg, D-20355 Hamburg, Germany, ${ }^{3}$ Institute of Physics, Polish Academy of Sciences, al. Lotników 32/46, PL-02 668 Warszawa, Poland, ${ }^{4}$ Institute of Theoretical Physics, University of Warsaw, ul. Hoża 69, PL 00681 Warszawa, Poland. †Present addresses: Department of Physics, Columbia University, New York, New York 10025, USA (U.W.); Solid State Physics Laboratory, ETH Zurich, Zurich 8093, Switzerland (W.W.). *e-mail:werner.wegscheider@phys.ethz.ch.
} 


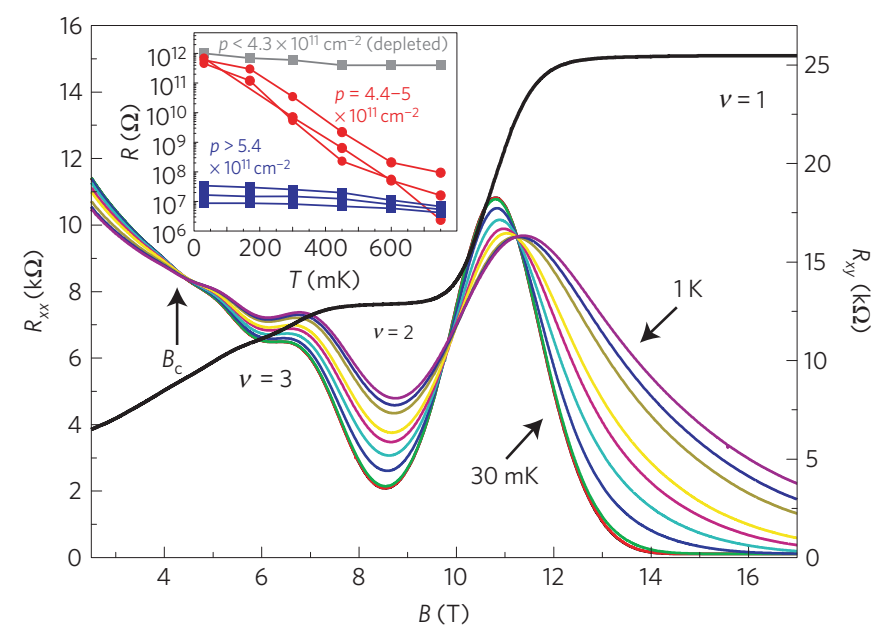

Figure 2 | Temperature dependence of longitudinal resistance of the inversely doped structure in high $B$ fields. The longitudinal resistance $R_{x x}$ along the [110] directions shows well-developed Shubnikov-de Haas oscillations with vanishing resistance at filling factor $v=1$. At low $B$ the system undergoes a quantum Hall insulator transition with a dramatic increase of $R_{x x}$ for all temperatures. The Hall resistance is shown for temperature $T=30 \mathrm{mK}$. Inset, Temperature dependence of the zero-field resistance $\left(U_{\text {bias }}=0.5 \mathrm{~V}\right)$ at various gate voltages, demonstrating the tunability from strongly to weakly localized with increasing carrier density.

Shubnikov-de Haas oscillations and quantum Hall plateaus in high fields. This clearly demonstrates the two-dimensional nature of the charge-carrier system and the absence of parallel conductance, a conclusion consistent with a sufficiently low Mn concentration to prevent an insulator-to-metal transition of Mn acceptors in the InAlAs barrier.

According to the experimental findings summarized in Figs 1a, 2 and 3, the 'inverted' structure (sample A) shows a dramatic and temperature-dependent increase of resistance in the zero-field range, indicating a strong hole localization under these conditions. The application of a perpendicular magnetic field leads to a colossal negative magnetoresistance, resulting eventually in the quantum Hall insulator transition at $B_{\mathrm{T}} \cong 4 \mathrm{~T}$, above which the longitudinal resistance decreases with decreasing temperature (Fig. 2). In this high-field range the quantum Hall effect emerges with a welldeveloped plateau and a corresponding zero-resistance state at low temperatures (Figs 1a and 2).

Because of the extremely high resistance values at low temperatures and magnetic fields, measurements in this region have been made in a two-terminal geometry by applying a constant voltage $U_{\text {bias }}$ to the sample and measuring the current $I$ according to $R=U_{\text {bias }} / I$. As depicted in Fig. 3a-c, field-induced hole delocalization is accompanied by resistance jumps over several orders of magnitude from above $10^{11}$ to below $10^{6} \Omega$, particularly abrupt for a relatively high bias voltage of $U_{\text {bias }}=0.5 \mathrm{~V}$ (Fig. $3 \mathrm{~b}, \mathrm{c}$ ), where the dependence $R\left(U_{\text {bias }}\right)$ is nonlinear at low temperatures (Fig. 3d). At the same time, the resistance shows evidence of a notable hysteretic behaviour when sweeping the magnetic field across zero, that is, the resistance at high and low $B$ is asymmetric with respect to zero field (Fig. $3 \mathrm{a}-\mathrm{c}$ ). As seen, the changes and jumps of the resistance as well as the hysteretic behaviour diminish and finally disappear above $\sim 0.6 \mathrm{~K}$.

According to results collected in Fig. 3c, hole accumulation by a negative top-gate voltage shifts the system away from the localization boundary to a region where the resistance is smaller and much less temperature dependent. In this regime, (abrupt) resistance changes vanish and hystereses are reduced. Depletion of the channel moves both the magnetic-field value where the resistance drops $\left(B_{\text {jump }}\right)$ and the resistance at $B>B_{\text {jump }}$ to higher values.
Importantly, only the perpendicular component of the external magnetic field matters for switching the conductivity state. In the inset to Fig. 3d, $B_{\text {jump }}$ and its sample normal component $\left(B_{z}\right)$ are plotted for several angles between the sample normal $\left(0^{\circ}\right)$ and the field direction. The switching occurs at the same value of $B_{z}$ for all angles.

We note that, according to our results obtained for samples grown at various $\mathrm{Mn}$ fluxes, the relevant quantity influencing the resistance is not the absolute value of the hole density but rather the ratio of the hole density to Mn concentration. This is demonstrated in Fig. 3e, where the relevant data for an 'inverted' structure (sample B) with about three times higher Mn density and more than doubled two-dimensional hole density $p$ are shown. The extraordinary magnetoresistance behaviour in this sample is virtually identical to that discussed above.

To interpret these findings we note that, as expected from the theory of the Anderson-Mott localization, when the carrier density is very low the holes are strongly localized by the parent acceptors, independent of the strength of the external magnetic field. If, in turn, the hole density is sufficiently high, in particular greater than the $\mathrm{Mn}$ concentration, owing to the combined effect of many-body screening, large kinetic energy and the extended character of wavefunctions corresponding to the upper Hubbard band of the acceptor states, the holes become delocalized, the effect visible in Figs 2 and 3c. Most interesting is the intermediate range of carrier densities, in which the metallic-like conductivity is observed at high magnetic field, but a crossover to the strongly localized regime occurs when the magnetic field decreases. This phenomenon, accounting for the celebrated colossal negative magnetoresistance ${ }^{1-9}$, occurs if the spins do not show long-range ferromagnetic order. As reviewed elsewhere ${ }^{16}$, in such a case carrier localization is enhanced at low magnetic fields by two effects: (1) spin-disorder scattering on randomly oriented preformed ferromagnetic bubbles brought about by spatial fluctuations in the local density of carrier states near the Anderson-Mott localization, and (2) the decrease of the kinetic energy associated with the carrier redistribution over the two spin subbands. Obviously, the redistribution of holes between the relevant subbands depends strongly on the magnetization direction ${ }^{4,17}$, and is accompanied by an increasing contribution of light holes to the effective mass of carriers at the Fermi level, which enhances dramatically the field-induced hole delocalization ${ }^{4}$.

This scenario implies that no spontaneous long-range ferromagnetic order develops in the InAs channel hosting the two-dimensional hole gas (2DHG) in the relevant temperature range. This assumption seems to be true, as for the QWs containing weakly localized holes with the determined effective mass of $m^{*}=0.16 m_{o}$ (see Methods) the expected Curie temperature is ${ }^{14}$ $T_{\mathrm{C}}<20 \mathrm{mK}$ for the Mn content indicated by the secondary-ion mass spectroscopy measurements, $x<10^{-4}$.

We now make evident that the high-resistance state, resistance jumps and hysteresis are due to the interplay of two bistabilities. First, following a recent theory ${ }^{10}$, we address the consequences of bistability due to overheating of the hole gas in the region of high electric fields: owing to a strong temperature dependence of the resistance in weak magnetic fields and at fixed high bias voltage, the system of Mn spins and carriers is either in the overheated (low-resistance) state or in the much less heated high-resistance state. The starting point to show the significance of overheating at non-zero bias voltages is the heat-balance equation,

$$
U_{\text {bias }}^{2} /\left[R\left(T_{h}, B\right) L W\right]=F_{\mathrm{S}}\left(T_{h}\right)-F_{\mathrm{S}}\left(T_{\mathrm{s}}\right)
$$

where $F_{S}(T)$ is the energy loss rate per unit of the QW surface $L W$, determined by the coupling to acoustic phonons at the hole and substrate temperatures $T_{\mathrm{h}(\mathrm{s})}$, respectively ${ }^{10}$. The right-hand side of 


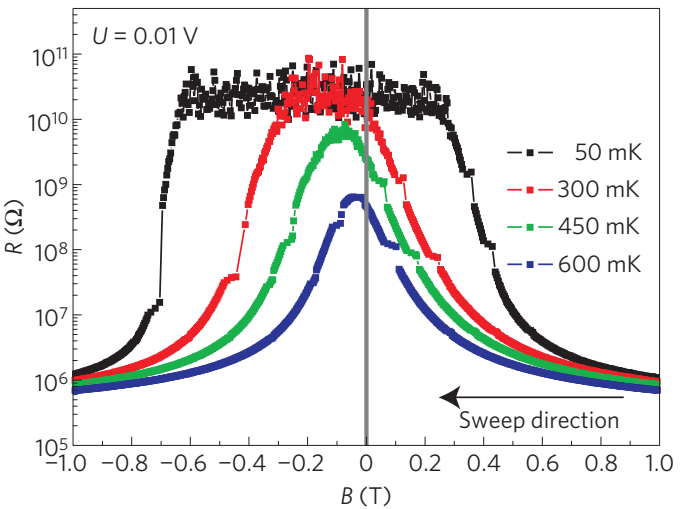

C

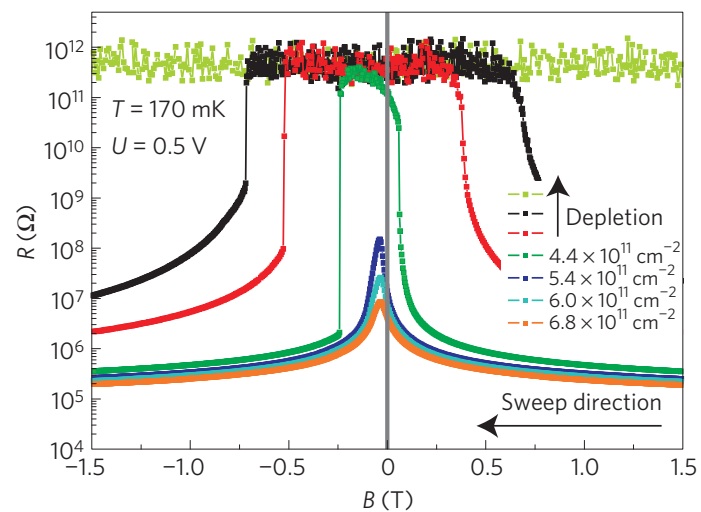

e

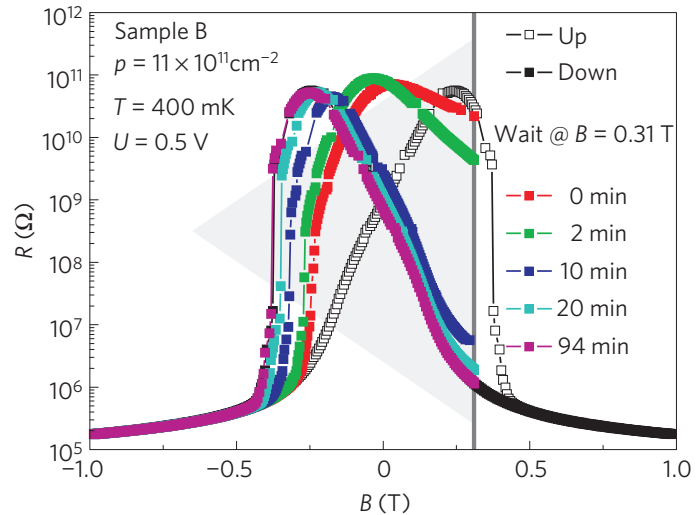

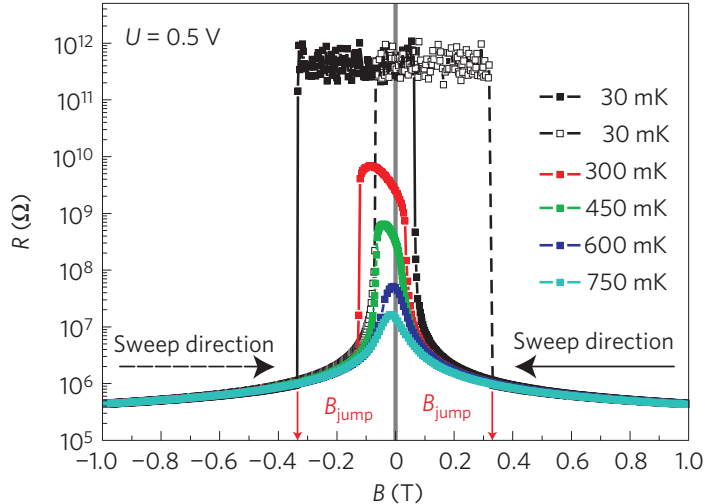

d
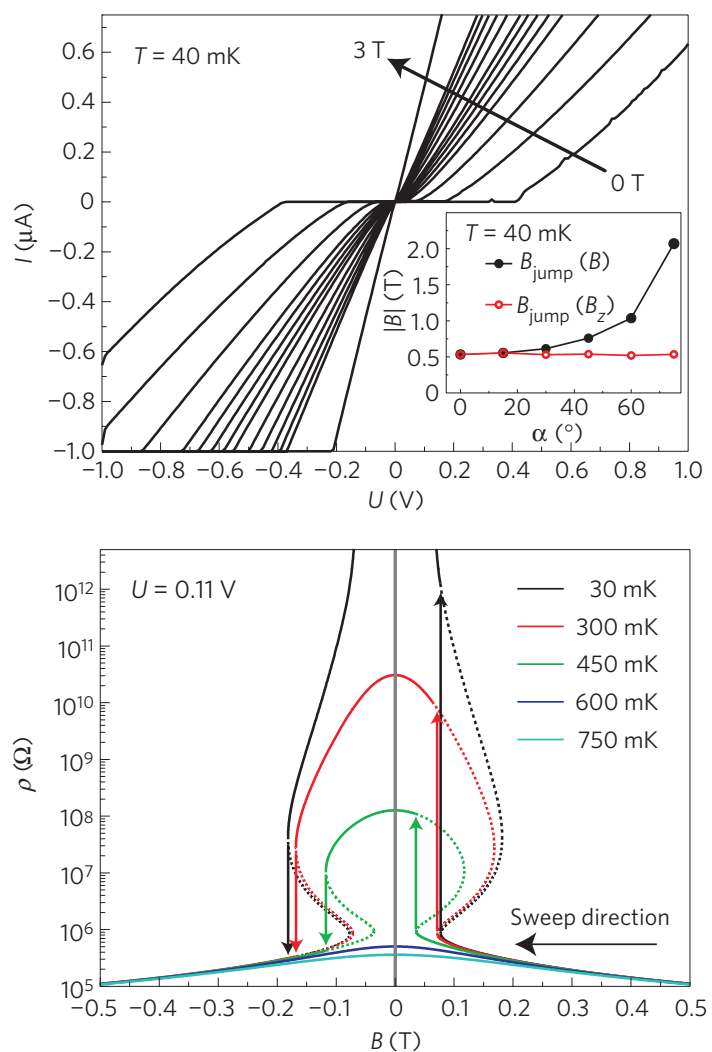

Figure 3 | Magnetic-field-driven hole delocalization in the inverted doped structure. a-f, Two-terminal resistance of the inverted structure-sample $A$ (a-d); sample B (e); computed (f). a,b, Magnetoresistance showing temperature-dependent hystereses and abrupt resistance drops for $U_{\text {bias }}=0.01 \mathrm{~V}$ (a) and $U_{\text {bias }}=0.5 \mathrm{~V}(\mathbf{b}) ; R_{\max }=10^{10} \Omega(\mathbf{a})$ and $R_{\max }=10^{12} \Omega(\mathbf{b})$ denotes an upper limit of the experimental set-up. c, Magnetoresistance at different carrier densities varied by the top-gate voltage at $T=170 \mathrm{mK}$. d, Current-voltage characteristics at $T=40 \mathrm{mK}$ for selected values of perpendicular magnetic field (sweep direction marked by an arrow) showing highly nonlinear and linear behaviour at low and high magnetic fields, respectively. The inset shows the magnitude of the total magnetic field corresponding to the abrupt resistance decrease, $B_{\text {jump }}$ (black), and the normal component of $B_{z}$ (red) as a function

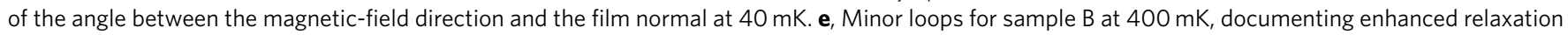
times. Upward $B$-field sweeps were stopped at $B=0.31 \mathrm{~T}$. Then, after different waiting times indicated in the figure, the magnetic field was swept down. Open black squares show a full up-sweep (after complete relaxation). $\mathbf{f}$, Computed resistance as a function of the magnetic field at various substrate temperatures for $L=0.9 \mathrm{~mm}$ and $U_{\text {bias }}=0.11 \mathrm{~V}$ for sample A. Positions of resistance jumps are marked by arrows.

equation (1) describes the energy flow from the hole bath to the phonon bath, provided by the Joule heating on the left-hand side. As the effects of the Mn spins on charge transport scale with magnetic susceptibility ${ }^{4,5,9}$ and the resistance $R(T)$ varies exponentially ${ }^{10}$ in this regime, (see Fig. 2 inset), we assume

$$
R(T, B)=R_{0} \exp \left(a\left[\mathrm{~d} B_{5 / 2}(T, B) / \mathrm{d} B\right] / T\right)^{\gamma}
$$

where $B_{5 / 2}(T, B)$ is the Brillouin function for $S=5 / 2 ; R_{0}, a$ and $\gamma$ are parameters determined by fitting $R(T, B=0)$ to the experimental zero-field resistance.
As shown in Fig. 3f, the model, developed with no further fitting parameters (see Supplementary Information), describes the presence of the resistance jumps, corresponding to the transition from the high-resistance state to the overheated low-resistance state. However, as the potential drop and carrier cooling at the contacts are ignored ${ }^{10}$, the range of $U_{\text {bias }}, T$ and $B$ where the jumps occur is lower than in the experiment.

Although the above picture reproduces the jumps occurring at different $B$ fields for up- and down-sweeps, the model fails to describe the hysteresis at low $B$, as the calculated resistance is symmetric with respect to $B$, in contrast to experiment. 
Actually, according to data in Fig. $3 a$ and direct magnetization measurements ${ }^{18}$, the magnetic hystereses persist even for $U_{\text {bias }} \rightarrow 0$. At the same time, minor loops with magnetic field stopped for different time durations (Fig. 3e) point to a finite relaxation time of several minutes, implying the absence of ferromagnetic order.

We shall now demonstrate that the presence of magnetic hysteresis without long-range magnetic order follows from specific properties of our system. Unlike (Ga,Mn)As (refs 19,20), compressively strained InAs QWs show a large energy separation between the heavy- and the light-hole subbands, which reduces the admixture of $j_{z}= \pm 1 / 2$ states to the wavefunctions of the holes residing in the ground-state subband, $j_{z}= \pm 3 / 2$ (with the $z$-axis perpendicular to the QW plane). Accordingly, the heavy-hole intraband matrix elements of $j_{x}$ and $j_{y}$ are very small, so the system is immune to an inplane magnetic field, in agreement with the experimental findings shown in Fig. 3d. This also means the appearance of large anisotropy energy barriers for reversing the magnetization direction of the preformed ferromagnetic bubbles. The corresponding relaxation time will grow exponentially with the number of holes contributing to the bubble, resulting in superparamagnetic-like metastabilities.

We expect the presence of a metastable behaviour in weak magnetic fields, even if the holes are bound by individual $\mathrm{Mn}$ acceptors. In this limit, the hole spin is coupled to the parent $\mathrm{Mn}$ acceptor by a strong antiferromagnetic exchange interaction ${ }^{12}$, which in the present case assumes the Ising form $H=-\varepsilon_{\mathrm{c}} j_{z} S_{z}$, where $\varepsilon_{\mathrm{c}}=-(\beta / 3)|\psi(0)|^{2}$. Here $\beta=-0.054 \mathrm{eV} \mathrm{nm}^{3}$ is the $p-d$ exchange integral and $\psi(0)$ is the value of the acceptor envelope function at the $\mathrm{Mn}$ ion. The relaxation time $\tau_{\mathrm{s}}$ between the two relevant heavy-hole $j_{z}= \pm 3 / 2$ states is rather long, presumably in a millisecond to microsecond range ${ }^{21,22}$. A somewhat shorter relaxation time is expected for $\mathrm{Mn}$ spins in the relevant range of concentrations ${ }^{23,24}$. Under these conditions a direct hole-spin relaxation is possible through a flip-flop, $j_{z} \rightarrow-j_{z},-S_{z} \rightarrow S_{z}$, process. However, the corresponding rate is expected to be rather small, particularly at low temperatures, where it would involve particularly slow transitions between $\pm 5 / 2$ states of the ion in the orbital singlet state. Furthermore, the $p-d$ coupling removes the degeneracy of the Mn spin states, which reduces the role of nuclear magnetic moments in the spin relaxation ${ }^{24,25}$.

In this situation, spin relaxation of holes towards thermalequilibrium values of $\left\langle j_{z}\right\rangle$ and, then, $\left\langle S_{z}\right\rangle$, leading to the corresponding values of resistance, proceeds primarily through high-energy intermediate states determined by the magnitude of $S_{z}$. The presence of the magnetic anisotropy barrier $E_{\mathrm{a}}$ elongates the relaxation time $\tau_{\mathrm{s}}$ of the hole spin by $\exp \left(E_{\mathrm{a}} / k_{\mathrm{B}} T\right)$. According to the quantitative model presented in Supplementary Information, the barrier vanishes in the magnetic field $B_{\mathrm{c}} \approx \varepsilon_{\mathrm{c}} S /\left(2 \kappa \mu_{\mathrm{B}}\right)$ and attains the value $E_{\mathrm{a}} \approx \varepsilon_{\mathrm{c}} S j$ at $B=0$ and $T \rightarrow 0$, where for the QW in question the Luttinger parameter is $\kappa=7.53$ (ref. 26) and $\varepsilon_{\mathrm{c}}$ varies between 0 and $\sim 0.12 \mathrm{meV}$ for the $\mathrm{Mn}$ acceptors at the QW edge and centre, respectively.

We find that for $\varepsilon_{\mathrm{c}}=0.12 \mathrm{meV}$ and $\tau_{\mathrm{s}}=1 \mathrm{~ms} \tau$ reaches $100 \mathrm{~s}$ at $0.49 \mathrm{~K}$, the time during which the magnetic field changes by $\sim 0.4 \mathrm{~T}$ in our experiment. This explains the hysteretic behaviour and resistance relaxation in the way seen in millikelvin studies of molecular magnets ${ }^{27,28}$ and individual rare-earth ions ${ }^{25}$. In agreement with this evaluation, hystereses disappear above $\sim 0.6 \mathrm{~K}$. At the same time, the barrier is expected to vanish at $B_{\mathrm{c}}=0.34 \mathrm{~T}$, in accordance with the magnitudes of the apparent coercive fields at $T \rightarrow 0$. When the hole concentration increases, more and more holes occupy states with smaller values of $\varepsilon_{\mathrm{c}}$, meaning that the barrier height, and thus the relaxation time and the width of the hystereses, diminishes, the effect visible in Fig. 3c. Moreover, the model invoking properties of individual acceptors rather than a collective action of many Mn ions explains why the observed behaviour scales with the ratio of the $\mathrm{Mn}$ to hole density and not with the Mn concentration.
In conclusion, our results demonstrate that the field-induced delocalization of holes in Mn-modulation-doped III-V QWs proceeds through an intermediate and previously unknown metastable insulator phase. Within our model the jumps result from hole overheating, whereas hysteresis stems from a large magnetic anisotropy of the heavy holes, coupled to the parent Mn acceptors by the strong $p-d$ exchange interaction. The slow spin relaxation of individual bound holes revealed here, appealing from the viewpoint of quantum information processing and storing, should also appear in the case of single $\mathrm{Mn}$ acceptors residing in InAs quantum dots ${ }^{29}$. Interestingly, it may compete with spin quantum tunnelling at sufficiently low temperatures ${ }^{25,27,28}$, provided that the decoherence rate of the complex is be smaller than the matrix element coupling the $S_{z}= \pm 5 / 2$ states.

\section{Methods}

The samples are patterned into standard L-shaped Hall bar geometries $(1,000 \mu \mathrm{m} \times 200 \mu \mathrm{m}$ and $200 \mu \mathrm{m} \times 40 \mu \mathrm{m})$ employing optical lithography and wet chemical etching. Ohmic contacts are prepared by soldering alloyed In $\mathrm{Zn}$ and annealed for $60 \mathrm{~s}$ at $300^{\circ} \mathrm{C}$. For gate electric-field-dependent measurements some samples are covered with a 130-nm-thick insulating parylene film and a thin $\mathrm{Ti} / \mathrm{Au}$ top-gate electrode. The measurements are carried out either in a ${ }^{4} \mathrm{He}$ bath cryostat or in a dilution refrigerator. Transport measurements in the low-resistance range (high magnetic fields and/or temperatures above $1.5 \mathrm{~K}$ ) are made using a standard low-frequency lock-in technique with operation currents of $100 \mathrm{nA}$. In the high-resistance state (low temperatures and low magnetic fields), a constant voltage $U_{\text {bias }}$ was applied, the current through the sample was monitored, and the two-terminal resistance was calculated as $R_{2 \text {-term }}=U_{\text {bias }} / I$. In the low- $B$, low- $T$ regime, the magnetotransport depends on the sweep rate, which was here set to $0.25 \mathrm{~T} \mathrm{~min}^{-1}$

The hole density $p$ of the 2DHG was determined from classical Hall resistance and confirmed by the period of Shubnikov-de Haas oscillations. The characteristic value for the 'normal' doped 2DHGs is $p=4.3 \times 10^{11} \mathrm{~cm}^{-2}$. The corresponding values for the 'inverted' doped 2DHGs are $p=4.4 \times 10^{11} \mathrm{~cm}^{-2}$ and $11 \times 10^{11} \mathrm{~cm}^{-2}$, for samples A and B, respectively. The values of the effective mass $m^{*}=0.16 m_{0}$ determined from the cyclotron resonance measurements ${ }^{30}$ for an 'inverted' doped sample is larger than expected for the band-edge in-plane hole mass of the ground-state subband in an infinitely deep InAs QW (ref. 31), but consistent with previous experimental studies ${ }^{32}$.

Received 4 June 2009; accepted 10 August 2010; published online 3 October 2010

\section{References}

1. Shapira, Y., Foner, S., Aggarwal, R. L. \& Reed, T. B. EuO. II. Dependence of the insulator-metal transition on magnetic order. Phys. Rev. B 8, 2316-2326 (1973).

2. von Molnar, S., Briggs, A., Flouquet, J. \& Remenyi, G. Electron localization in a magnetic semiconductor: $\mathrm{Gd}_{3-x} \mathrm{v}_{x} \mathrm{~S}_{4}$. Phys. Rev. Lett. 51, 706-709 (1983).

3. Dagotto, E., Hotta, T. \& Moreo, A. Colossal magnetoresistant materials: The key role of phase separation. Phys. Rep. 344, 1-153 (2001).

4. Wojtowicz, T., Dietl, T., Sawicki, M., Plesiewicz, W. \& Jaroszyński, J. Metal-insulator transition in semimagnetic semiconductors. Phys. Rev. Lett. 56, 2419-2422 (1986)

5. Terry, I., Penney, T., von Molnár, S. \& Becla, P. Low-temperature transport properties of $\mathrm{Cd}_{0.91} \mathrm{Mn}_{0.09} \mathrm{Te}: \mathrm{In}$ and evidence of a magnetic hard gap in the density of states. Phys. Rev. Lett. 69, 1800-1803 (1992).

6. Oiwa, A. et al. Giant negative magnetoresistance of (Ga,Mn)As/GaAs in the vicinity of a metal-insulator transition. Phys. Status Solidi 205, 167-171 (1998).

7. Smorchkova, I. P., Samarth, N., Kikkawa, J. M. \& Awschalom, D. D. Spin transport and localization in a magnetic two-dimensional electron gas. Phys. Rev. Lett. 78, 3571-3574 (1997).

8. Smorchkova, I. P., Samarth, N., Kikkawa, J. M. \& Awschalom, D. D. Giant magnetoresistance and quantum phase transitions in strongly localized magnetic two dimensional electron gases. Phys. Rev. B 58, R4238-R4241 (1998).

9. Jaroszyński, J. et al. Intermediate phase at the metal-insulator boundary in a magnetically doped two-dimensional electron system. Phys. Rev. B 76, 045322 (2007).

10. Altshuler, B. L., Kravtsov, V. E., Lerner, I. V. \& Aleiner, I. L. Jumps in current-voltage characteristics in disordered films. Phys. Rev. Lett. 102, 176803 (2009).

11. Wurstbauer, U. et al. Coexistence of ferromagnetism and quantum Hall-effect in Mn modulation-doped two-dimensional hole system. J. Cryst. Growth 311, 2160-2162 (2009).

12. Ohno, H., Munekata, H., Penney, T., von Molnár, S. \& Chang, L.L. Magnetotransport properties of p-type (In,Mn)As diluted magnetic III-V semiconductors. Phys. Rev. Lett. 68, 2664-2667 (1992). 
13. Bhattacharjee, A. K. \& Benoit à la Guillaume, C. Model for the Mn acceptor in GaAs. Solid State Commun. 113, 17-21 (2000).

14. Boukari, H. et al. Light and electric field control of ferromagnetism in magnetic quantum structures. Phys. Rev. Lett. 88, 207204 (2002).

15. Wurstbauer, U. \& Wegscheider, W. Magnetic ordering effects in a Mn-modulation-doped high mobility two-dimensional hole system. Phys. Rev. B 79, 155444 (2009).

16. Dietl, T. Interplay between carrier localization and magnetism in diluted magnetic and ferromagnetic semiconductors. J. Phys. Soc. Jpn 77, 031005 (2008).

17. Pappert, K. et al. Magnetization-switched metal-insulator transition in a (Ga,Mn)As tunnel device. Phys. Rev. Lett. 97, 186402 (2006).

18. Rupprecht, B. et al. Magnetism in a Mn modulation-doped InAs/InGaAs heterostructure with a two-dimensional hole system. J. Appl. Phys. 107, 093711 (2010).

19. Sheu, B. L. et al. Onset of ferromagnetism in low-doped $\mathrm{Ga}_{1-x} \mathrm{Mn}_{x}$ As. Phys. Rev. Lett. 99, 227205 (2007).

20. Myers, R. C. et al. Zero-field optical manipulation of magnetic ions in semiconductors. Nature Mater. 7, 203-208 (2008).

21. Gerardot, B. D. et al. Optical pumping of a single hole spin in a quantum dot. Nature 451, 441-444 (2007).

22. Heiss, D. et al. Observation of extremely slow hole spin relaxation in self-assembled quantum dots. Phys. Rev. B 76, 241306(R) (2007)

23. Dietl, T., Peyla, P., Grieshaber, W. \& Merle d'Aubigné, Y. Dynamics of spin organization in diluted magnetic semiconductors. Phys. Rev. Lett. 74, 474-477 (1995).

24. Goryca, M. et al. Magnetization dynamics down to a zero field in dilute (Cd,Mn)Te quantum wells. Phys. Rev. Lett. 102, 046408 (2009).

25. Giraud, R., Wernsdorfer, W., Tkachuk, A. M., Mailly, D. \& Barbara, B. Nuclear spin driven quantum relaxation in $\mathrm{LiY}_{0.998} \mathrm{Ho}_{0.002} \mathrm{~F}_{4}$. Phys. Rev. Lett. 87, 057203 (2001).

26. Sanders, G. D. et al. Electronic states and cyclotron resonance in n-type InMnAs. Phys. Rev. B 68, 165205 (2003).
27. Friedman, J. R., Sarachik, M. P., Tejada, J. \& Ziolo, R. Macroscopic measurement of resonant magnetization tunneling in high-spin molecules. Phys. Rev. Lett. 76, 3830-3833 (1996).

28. Thomas, L. et al. Macroscopic quantum tunnelling of magnetization in a single crystal of nanomagnets. Nature 383, 145-147 (1996).

29. Kudelski, A. et al. Optically probing the fine structure of a single $\mathrm{Mn}$ atom in an InAs quantum dot. Phys. Rev. Lett. 99, 247209 (2007).

30. Wurstbauer, U. et al. Anomalous magnetotransport and cyclotron resonance of high mobility magnetic 2 DHG in the quantum Hall regime. Physica E 42, 1022-1025 (2010)

31. Suemune, I. Band-edge hole mass in strained-quantum-well structures. Phys. Rev. B 43, 14099-14106 (1991).

32. Oettinger, K. et al. Dispersion relation, electron and hole effective masses in $\mathrm{In}_{x} \mathrm{Ga}_{1-x}$ As single quantum wells. J. Appl. Phys. 79, 1481-1485 (1996).

\section{Acknowledgements}

U.W., D.W. and W.W. acknowledge financial support from the Deutsche Forschungsgemeinschaft (DFG) through SFB 689, U.W. also acknowledges support from the Hamburg Cluster of Excellence on Nanospintronics, and T.D. and C.S. acknowledge financial support by the Humboldt Foundation and EC project FunDMS (ERC Advanced Grant).

\section{Author contributions}

Project planning, W.W., D.W.; structure growth and processing, U.W., W.W.; experiments and data analysis, U.W., W.W., D.W.; theory, C.Ś., T.D.; writing, T.D., U.W., W.W., D.W., C.Ś

\section{Additional information}

The authors declare no competing financial interests. Supplementary information accompanies this paper on www.nature.com/naturephysics. Reprints and permissions information is available online at http://npg.nature.com/reprintsandpermissions. Correspondence and requests for materials should be addressed to W.W. 